\title{
Studies on Reaction Conditions for Size-selective Photoetching of Cadmium Telluride Nanocrystals
}

\author{
Taro Uematsu,
}

\author{
aDepartment of Applied Chemistry, Graduate School of Engineering, Osaka University (Suita, Osaka 565-0871, \\ Japan) \\ bJapan Science and Technology Agency, CREST (Kawaguchi, Saitama 332-0012, Japan) \\ 'Deparatment of Crystalline Materials Science, Graduate School of Engineering, Nagoya University (Chikusa-ku, \\ Nagoya 464-8603, Japan)
}

Received September 4, 2009 ; Accepted December 15, 2009

\begin{abstract}
Effects of reaction conditions on size-selective photoetching of CdTe nanocrystals were presented focusing on electron mediator and wavelength of irradiating light. Methyl viologen, which was expected to work as an electron mediator of photo-induced oxygen reduction accelerating the photoetching, decreased significantly the rate of the reaction. The results are compared with the photoetching of CdS nanocrystals in terms of overpotential for oxygen reduction and adsorption of electron mediators on nanocrystals. Under optimized conditions, the photoetching can produce highly monodispersed nanocrystals having fluorescence peak of fwhm value as small as $23.5 \mathrm{~nm}$ around the wavelength region where CdTe nanocrystals are stably produced by the conventional synthesis. However, further blue shifts of irradiating light gradually increased the fluorescence fwhm value, and the exciton peaks became unclear as well. The results were considered by comparing with recently reported magic-sized nanoclusters.
\end{abstract}

Key Words : Size-selective Photoetching, Electron Mediator, CdTe Nanocrystals, Magic-sized Nanoclusters

\section{Introduction}

Semiconductor nanocrystals (NCs) are attracting intense attention because of their many desirable properties, such as large absorption coefficient, intense emission and durability when exposed to light irradiation. ${ }^{1-7)}$ Their properties as semiconductors make them absorb wide wavelength range of light that is shorter than the bandgap, enabling multicolored emission by exposing to a single light source. In fact, their energy bands are slightly discrete compared to those of bulk semiconductors due to the spatially confined electron-hole pairs called excitons. The absorption maxima concerning excitons, exciton peaks, are the indicator of good surface modification of nanocrystals. The quantum size effect, which increases bandgap as the size of the NC decreases, are one of the most remarkable properties of semiconductor NCs. ${ }^{8-12)}$ Therefore, the size of the NCs is very important factor determining their optical properties; i.e., absorption and emission spectra blue-shifts as the size of the NCs decrease.

In the conventional synthetic procedures, the size of $\mathrm{NCs}$ is controlled by heating the NC colloidal solution. A mean NCs diameter increases as they are heated in the solution, at the expense of smaller particles or excess monomer. ${ }^{13-15)}$ The former is called Ostwald ripening process where size distribution becomes wider as the reaction time elapse, while the latter to some extent narrows the size distribution during the reaction. In any cases, conventional synthetic methods cannot generate highly monodispersed NCs due to the inhomogeneity of the reactions. As far as we know, there is only a method to obtain monodispersed NCs solution by the post-treatment so-called size-selective precipitation, which utilizes the differences in solubility between different-sized particles. ${ }^{4,16,17)}$ However, if one will to obtain monodispersed NCs having a certain emission wavelength, the product yield end up with very low, i.e., the inverse of the number of separated fractions.

Recently we developed methods to obtain highly monodispersed $\mathrm{NCs}$ of $\mathrm{CdS},{ }^{18-22} \mathrm{CdSe}^{23)}$ and $\mathrm{CdTe}^{24)}$ by using photocorrosion. The photocorrosion occurs by positive holes after removing electrons from excited semiconductor NCs. Then, the quantum size effect increases the bandgap of NCs with the blue shifts of the optical properties. When the monochromatic light is used, photoetching proceeds until the bandgap becomes equal to the irradiating energy, giving highly monodispersed $\mathrm{NCs}$ with their bandgap corresponding to irradiating energy.

In the present study, factors determining efficiency of the photoetching of CdTe NCs were studied. Effects of solution composition, especially, the role of electron mediator were investigated, and they were compared with the photoetching of CdS NCs. Under the optimized reaction conditions the photoetching produced highlymonodispersed CdTe NCs having PL corresponding to irradiating wavelength with fwhm of PL peak as small as $23.5 \mathrm{~nm}$. When the wavelength of the irradiating light was further blue shifted, the resulting CdTe NCs emitted nearly white light with their exciton peak became unclear. The results were compared with the recently developed fluorescent magic-sized nanoclusters of cadmium chalcogenide $\mathrm{NCs} .{ }^{25-37)}$ 


\section{1 Chemicals}

\section{Experimental}

We purchased cadmium chloride, sodium borohydride, thioglycolic acid (TGA), and sodium hydroxide from Wako Pure Chemicals Industries and used them without further purification. Methyl viologen dichloride was purchased from TCI. Te powder (99.8\%, 200 mesh) was purchased from Aldrich. Water used in this study was purified by Milli-Q gradient A10 (Millipore, $18.2 \mathrm{M} \Omega \mathrm{cm}$ ).

\section{2 Synthesis of CdTe nanocrystals}

CdTe nanocrystals were synthesized following Weller et al. with some modification. Briefly, tellurium powder was reacted with sodium borohydride in aqueous solution at $0{ }^{\circ} \mathrm{C}$; after $8 \mathrm{~h}$ a pale red colored NaHTe solution was obtained. In another flask, cadmium chloride and TGA were dissolved in $125 \mathrm{~mL}$ water, and the solution's $\mathrm{pH}$ was adjusted to 9 by the addition of $1 \mathrm{M} \mathrm{NaOH}$. After de-aeration, the prepared NaHTe solution was added with agitation to obtain the precursor of the nanocrystals. The molecular ratio of $\mathrm{Cd}$ : TGA : Te was $1: 1.8$ : 0.25 . The solution was refluxed for several hours until the nanocrystals grew to the desired particle size. The quality of nanocrystals was checked with UV-Vis and fluorescence spectra using a photodiode array spectrometer (Shimadzu, MultiSpec-1500) and a fluorospectrometer (Hitachi, F-4500). We measured quantum yield using a diode-array spectrometer equipped with an integrating sphere (Hamamatsu, PMA-12).

\section{3 Size-selective photoetching}

The CdTe NC colloidal solution was diluted by a factor of 20 with $\mathrm{NaOH}$ or $\mathrm{KOH}$ aqueous solution of different concentrations and $\mathrm{pH}$ values. Some of the samples contained $1 \mathrm{mM}$ of methyl viologen as an electron mediator. An aliquot of $3 \mathrm{~mL}$ was put into a quartz cell $(1 \times 1 \times 4$ $\mathrm{cm}$ ) and oxygen was bubbled into the solution for $10 \mathrm{~min}$ prior to photoetching. The solution was irradiated with monochromatic light from an $\mathrm{Hg}$ lamp through a monochrometer (Bunkoh-Keiki, KX-025). The power of the light was $\sim 10 \mathrm{~mW} / \mathrm{cm}^{2}$ in the wavelength region used for the photoetching (500-600 nm), and the area of irradiation was fixed to $\sim 2 \mathrm{~cm}^{2}$. The solution was stirred continuously during the photoetching process. Absorption and fluorescence spectra were measured intermittently during the course of photoetching. Irradiation was continued until a constant absorption spectrum was obtained.

\section{Results and Discussion}

\section{1 Effects of electron mediators}

Figure 1(a) shows changes in the absorption spectra as a function of duration time of photoetching without methyl viologen. The photoetching is a photo-oxidation of $\mathrm{NC}$ itself by the positive holes, after removing electrons from the photo-excited NC. Optimization of reaction conditions revealed that the photoetching of CdTe proceeded under strong alkaline conditions $(\mathrm{pH}>13.5)$, in which photo-oxidized species become soluble in the forms of $\mathrm{TeO}_{4}{ }^{2-}, \mathrm{TeO}_{3}{ }^{2-}$ and $\mathrm{HCdO}_{2}{ }^{-24)}$

The photoetching of CdS NCs in the presence of methyl viologen was three orders of magnitude faster
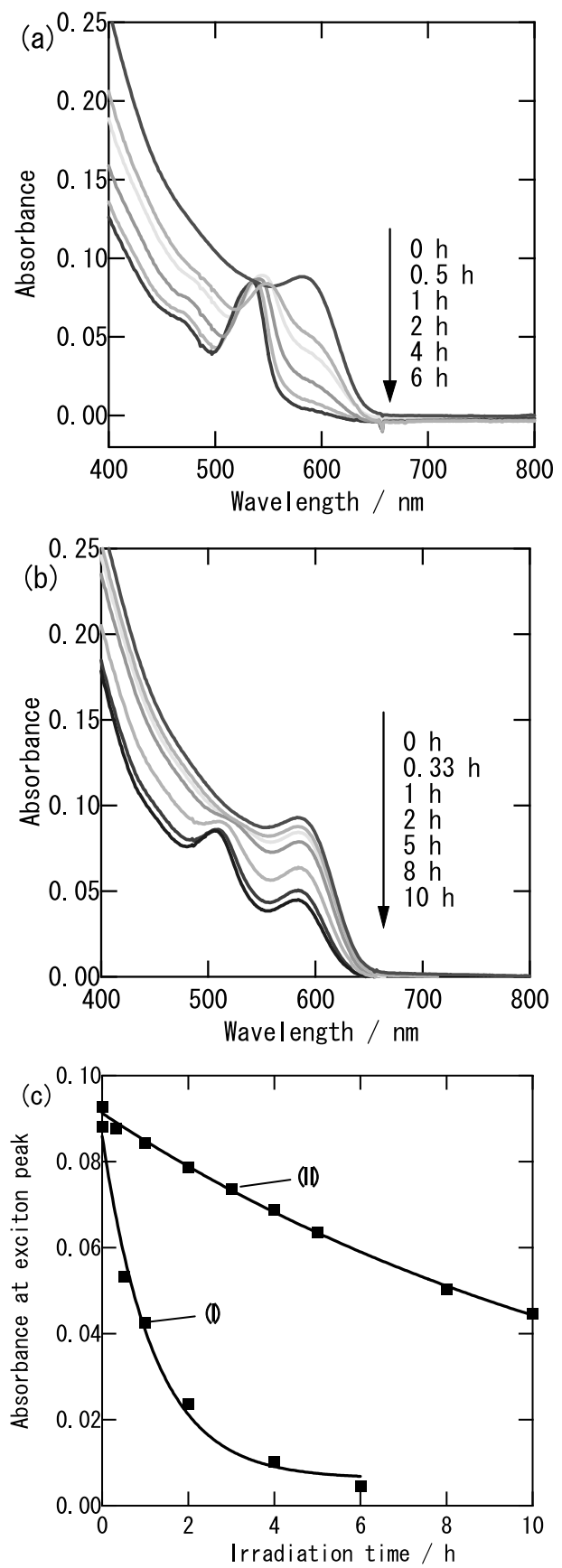

Fig. 1 Absorption spectra during the photoetching in the absence (a) and in the presence (b) of methyl viologen (1 $\mathrm{mM}$ ), and plots for absorbance at the exciton peak as a function of duration time of the photoetching; without (I) and with (II) electron mediator (c). The $\mathrm{pH}$ of the solution was 13.5, and the irradiation wavelength was $580 \mathrm{~nm}$.

than that without methyl viologen. ${ }^{19)}$ Therefore, we expected the addition of methyl viologen promotes the rate of the photoetching of CdTe. Figure 1(b) shows spectra of the same reaction as Fig. 1(a) but in the presence of $1 \mathrm{mM}$ methyl viologen. Unexpectedly, such effect was not observed; on the contrary, the reaction rate was slowed down by the addition of methyl viologen. Changes in the absorption at the wavelength of the original exciton peak were displayed in Fig. 1(c). Apparently, the reaction rate was lower by a factor of 10 in the pres- 
ence of methyl viologen. The large difference in the effects of electron mediator was discussed from two points: the difference in absolute band-edge potentials between CdS and CdTe, and the specific adsorption of methyl viologen on the surface of TGA-CdTe NCs.

Photoetching rate are considered to rely on the efficient removal of the electrons from photo-excited NCs. In the cases of hexametaphosphate-capped CdS (HMP-CdS) $\mathrm{NC}$, the edge of conduction band was expected to be -1.0 to -1.2 volts vs NHE dependent on its size, which was estimated from flatband potential of bulk CdS and sizequantization effects. ${ }^{38)}$ However, taking account of the fact that oxygen reduction on metal and semiconducting electrodes generally needs large overpotentials, these potentials are not negative enough for the sufficient removal of electrons from NCs by dissolved oxygen. On the other hand, in the case of CdTe NCs, the lower edge of the conduction band of the thiol-stabilized CdTe NCs was investigated by the pulse radiolysis experiment to be -2.0 to -2.4 volts dependent on the particle size. ${ }^{39)}$ Thus, the oxygen reduction, which is for instance -0.12 $\mathrm{V}$ for two electron reduction at $\mathrm{pH}=13.5$, occurred effectively without mediators, giving relatively faster photoetching rate compared with CdS NCs that inevitably need electron mediators. ${ }^{19)}$

The above mentioned largeness of overpotential cannot explain the decrease in the photoetching rate in the presence of methyl viologen. Here, we considered the possibility of adsorption of methyl viologen onto the $\mathrm{NCs}$ ' surface. Figure 2 shows the quenching of photoluminescence from TGA-CdTe by the addition of methyl viologen. The inset shows the Stern-Volmer plots, giving coefficients $\left(K_{\mathrm{SV}}\right)$ of $5.4 \times 10^{6} \mathrm{M}^{-1}$. This value is two to three orders of magnitude larger than $K_{\mathrm{Sv}}$ that reported for organic fluorophores and quenchers under diffusion mediated photoinduced electron transfer. Therefore, it is natural to consider that methyl viologen is adsorbed on the NCs, although they are not covalently bonded. Considering large potential gap between the lower conduction band edge of TGA-CdTe NC and methyl viologen, the electron transfer rate is expected to be much

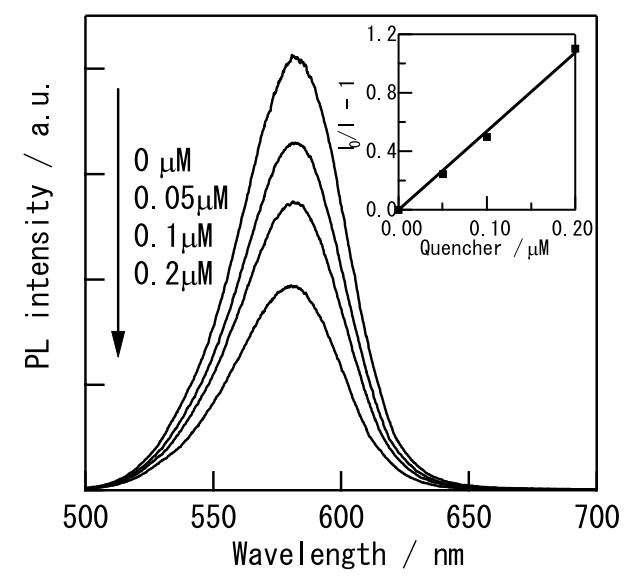

Fig. 2 Emission quench of CdTe NCs by methyl viologen. The inset is the corresponding Stern-Volmer plots $(\mathrm{Ex}=$ $460 \mathrm{~nm})$. faster than diffusion rate; and the quenching occurred through methyl viologen that is adsorbed on the NCs. Although quenching of HMP-CdS NCs were also reported to be caused by adsorbed methyl viologen, its $K_{\mathrm{SV}}$ value $\left(2.1 \times 10^{5} \mathrm{M}^{-1}\right)$ was $c a .25$ times smaller than that obtained in the present study for TGA-CdTe, indicating that the adsorption coefficient of methyl viologen on TGA-CdTe is much larger than that on HMP-CdS. ${ }^{40)}$

\section{2 Blue limit of photoetching}

One of the interests concerning size-selective photoetching is how small we can prepare the CdTe NCs. ${ }^{20}$ While the photoetching produced highly-monodispersed CdTe NCs with emission fwhm value as small as 23.5 $\mathrm{nm}$, the fwhm became larger by the irradiation less than $560 \mathrm{~nm}^{24)}$ Figure 3 shows the UV-Vis (a) and PL (b) spectra of TGA-CdTe NCs photoetched with various wavelengths, from 540 to $500 \mathrm{~nm}$. The exciton peak, which was clearly observed when irradiated with 540 $\mathrm{nm}$ light, became unclear as the further blue shifts of the irradiating light, while it shifted steadily from $499 \mathrm{~nm}$ (irradiation at $540 \mathrm{~nm}$ ) to $440 \mathrm{~nm}(500 \mathrm{~nm})$. The PL also decreased significantly as the extinction of the exciton peaks, leaving longer wavelength component. The QY decreased from $45 \%$ to $5.8 \%$, when the irradiation wavelength was changed from $580 \mathrm{~nm}$ to $500 \mathrm{~nm}$. Figure 4 shows the PL spectrum of TGA-CdTe photoetched with $500 \mathrm{~nm}$ light. It had a peak at $503 \mathrm{~nm}$, but the color of
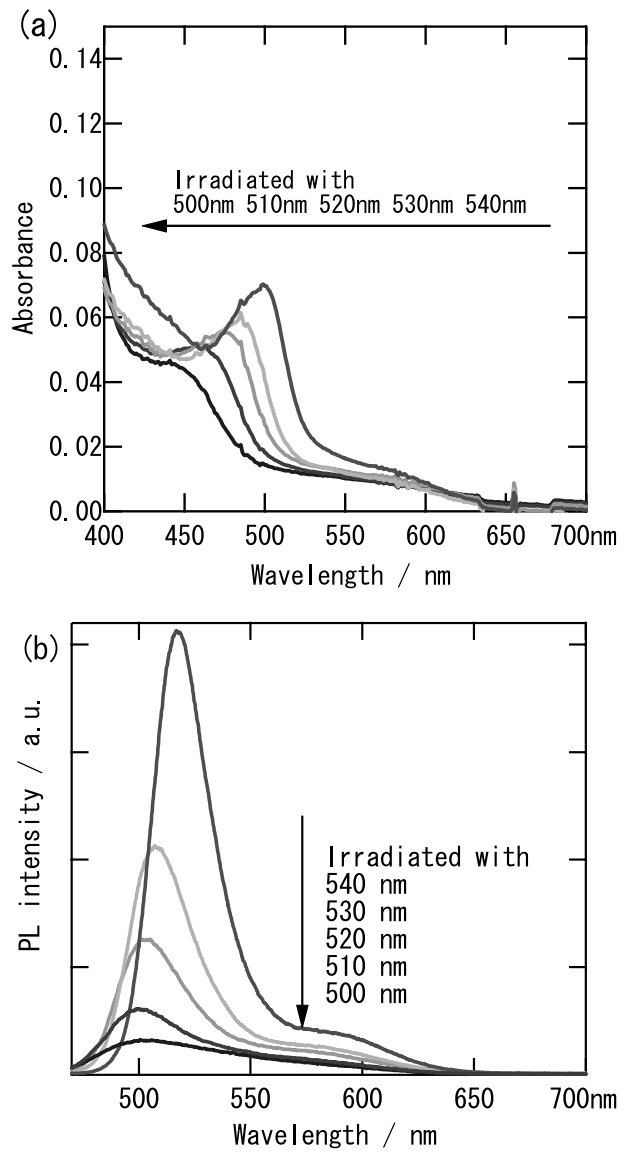

Fig. 3 Absorption (a) and PL (b) spectra $(\mathrm{Ex}=460 \mathrm{~nm})$ after the photoetching with irradiating wavelength less than $540 \mathrm{~nm}$. 


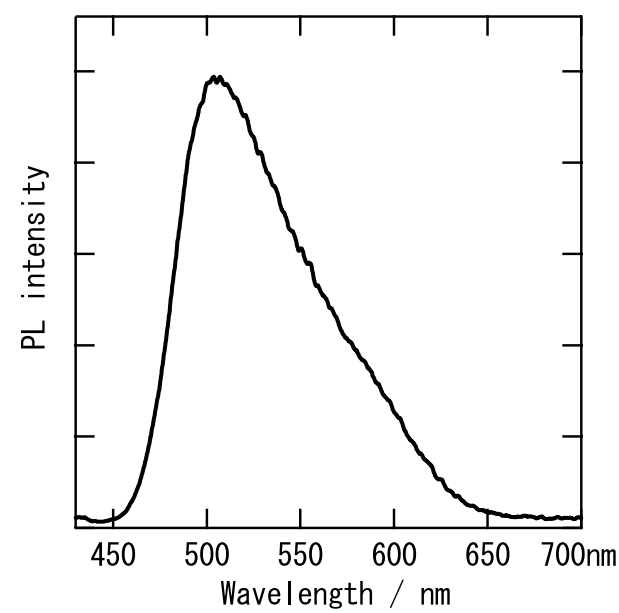

Fig. 4 PL spectrum of TGA-CdTe NCs photoetched with $500 \mathrm{~nm}$ irradiation. Excitation wavelength is $420 \mathrm{~nm}$.

emission when exposed to UV-light was almost white, as it can be expected from large fwhm of the PL $(84 \mathrm{~nm})$.

Although the quantum size effect steadily blue-shift the optical properties of semiconductor NCs, small limit until which particles can exist stably is known to exist. Some representative studies were done on very small particles called magic-sized clusters ${ }^{20,25-37)}$ which are thermodynamically stable small clusters having electronic closed-shell structures. They are considered to be generated in the early stage of the synthetic reactions of semiconductor NCs, and in some cases were preferentially synthesized and isolated under very strictly controlled reaction conditions. Some of the studies about magicsized clusters reported white-light emission originating from the increase in the surface trap sites. ${ }^{30-32)} \mathrm{We}$ also observed broad-band emission from the CdTe NCs photoetched with $500 \mathrm{~nm}$ light, and the wavelength of the exciton peak $(440 \mathrm{~nm})$ was comparable with magic-sized CdTe clusters synthesized by Chikan et al. ${ }^{28,31)}$ However, the exciton peak was not as clear as their reports, indicating that the resulting NCs are imperfect having many trap sites that recombine excitons. We speculated that the instability of these particles was due to the continuous reaction of photoetching, which lower significantly the stability of NCs deviated from magic-sizes, resulting in the increase in the surface traps and/or instant decomposition of particles themselves. The result that the photoetching of CdTe NCs to the diameter close to the magic-size is unfavorable coincided with our previous report of preparation of ultrasmall CdS NCs by the photoetching. ${ }^{20)}$

\section{Conclusion}

We investigated the reaction conditions for the photoetching of CdTe. In terms of solution composition, effects of electron mediator on size-selective photoetching were studied for CdTe NCs and they were compared with the previous results for CdS NCs. Addition of methyl viologen to the CdTe NCs solution during the photoetching significantly slowed down the reaction rate, while it accelerated about 3 orders of magnitude the pho- toetching rate of CdS NCs. These differences were due to the strong adsorption of methyl viologen on the CdTe NCs surface originating from large valency of TGA molecules densely bound to CdTe. Thus, methyl viologen served as a trap site of electrons, rather than the electron mediator. Under the optimized reaction conditions, blue limit of irradiating light for the photoetching was investigated. Although the photoetching of CdTe NCs produced highly-monodispersed particles with the fwhm of the PL peak as small as $23.5 \mathrm{~nm}$, further blue shifts of irradiating light lowered the monochromaticity. Furthermore, the exciton peak became unclear when its wavelength reached to that of magic-sized clusters which were recently reported by the precise control of the reaction conditions. This is due to the mismatch between the photoetching that is intrinsically the continuous reaction and the nature of magic-sized clusters that need preferential sizes to be stabilized.

\section{Acknowledgement}

This research was financially supported by Grant-inAid for Scientific Research (18201022) from Japan Society for the Promotion of Science (JSPS) and Grant-inAid for Scientific Research in Priority Areas "Science of Ionic Liquids" from the Ministry of Education, Culture, Sports, Science and Technology (MEXT). One of the authors (T. U.) expresses his gratitude to a Grant-in-Aid for JSPS Fellows and to the Global Center of Excellence (COE) Program "Global Education and Research Center for Bio-Environmental Chemistry" of Osaka University.

\section{References}

1) C. B. Murray, C. R. Kagan, and M. G. Bawendi, Annu. Rev. Mater. Sci., 30, 545 (2000).

2) A. P. Alivisatos, W. Gu, and C. Larabell, Annu. Rev. Biomed. Eng., 7, 55 (2005).

3) P. V. Kamat, Journal of Physical Chemistry C, 111, 2834 (2007).

4) N. Gaponik, D. V. Talapin, A. L. Rogach, K. Hoppe, E. V. Shevchenko, A. Kornowski, A. Eychmüller, and H. Weller, J. Phys. Chem. B, 106, 7177 (2002).

5) L. Qu and X. Peng, J. Am. Chem. Soc., 124, 2049 (2002).

6) P. Yu, K. Zhu, A. G. Norman, S. Ferrere, A. J. Frank, and A. J. Nozik, J. Phys. Chem. B, 110, 25451 (2006).

7) W. C. W. Chan and S. M. Nie, Science, 281, 2016 (1998).

8) S. V. Gaponenko, Optical Properties of Semiconductor Nanocrystals, Cambridge University Press, Cambridge, (1998).

9) M. G. Bawendi, W. L. Wilson, L. Rothberg, P. J. Carroll, T. M. Jedju, M. L. Steigerwald, and L. E. Brus, Phys. Rev. Lett., 65, 1623 (1990).

10) M. G. Bawendi, M. L. Steigerwald, and L. E. Brus, Annu. Rev. Phys. Chem., 41, 477 (1990).

11) A. P. Alivisatos, J. Phys. Chem., 100, 13226 (1996).

12) A. P. Alivisatos, Science, 271, 933 (1996).

13) X. Peng, J. Wickham, and A. P. Alivisatos, J. Am. Chem. Soc., 120, 5343 (1998).

14) D. V. Talapin, A. L. Rogach, E. V. Shevchenko, A. Kornowski, M. Haase, and H. Weller, J. Am. Chem. Soc., 124, 5782 (2002).

15) D. V. Talapin, A. L. Rogach, M. Haase, and H. Weller, J. 
Phys. Chem. B, 105, 12278 (2001).

16) C. B. Murray, D. J. Norris, and M. G. Bawendi, J. Am. Chem. Soc., 115, 8706 (1993).

17) A. Chemseddine and H. Weller, Ber. Bunsenges. Phys. Chem., 97, 636 (1993).

18) H. Matsumoto, T. Sakata, H. Mori, and H. Yoneyama, Chem. Lett., 595 (1995).

19) H. Matsumoto, T. Sakata, H. Mori, and H. Yoneyama, J. Phys. Chem., 100, 13781 (1996).

20) T. Torimoto, H. Kontani, Y. Shibutani, S. Kuwabata, T. Sakata, H. Mori, and H. Yoneyama, J. Phys. Chem. B, 105, 6838 (2001).

21) S. Kuwabata, K. Ueda-Sarson, and T. Torimoto, Chem. Lett., 33, 1344 (2004).

22) K. Sato, S. Kojima, S. Hattori, T. Chiba, K. Ueda-Sarson, T. Torimoto, Y. Tachibana, and S. Kuwabata, Nanotechnology, 18, (2007).

23) T. Torimoto, S. Y. Murakami, M. Sakuraoka, K. Iwasaki, K. I. Okazaki, T. Shibayama, and B. Ohtani, J. Phys. Chem. B, 110, 13314 (2006).

24) T. Uematsu, H. Kitajima, T. Kohma, T. Torimoto, Y. Tachibana, and S. Kuwabata, Nanotechnology, 20, 215302 (2009).

25) C. Tuinenga, J. Jasinski, T. Iwamoto, and V. Chikan, ACS Nano, 2, 1411 (2008).

26) J. Ouyang, M. B. Zaman, F. J. Yan, D. Johnston, G. Li, X. Wu, D. Leek, C. I. Ratcliffe, J. A. Ripmeester, and K. Yu, Journal of Physical Chemistry C, 112, 13805 (2008).

27) C. M. Evans, L. Guo, J. J. Peterson, S. MaccagnanoZacher, and T. D. Krauss, Nano Lett., 8, 2896 (2008).

28) P. Dagtepe and V. Chikan, J. Phys. Chem. A, 112, 9304
(2008).

29) S. Kudera, M. Zanella, C. Giannini, A. Rizzo, Y. Li, G. Gigli, R. Cingolani, G. Ciccarella, W. Spahl, W. J. Parak, and L. Manna, Adv. Mater., 19, 548 (2007).

30) Q. Dai, D. Li, J. Chang, Y. Song, S. Kan, H. Chen, B. Zou, W. Xu, S. Xu, B. Liu, and G. Zou, Nanotechnology, 18, (2007).

31) P. Dagtepe, V. Chikan, J. Jasinski, and V. J. Leppert, Journal of Physical Chemistry C, 111, 14977 (2007).

32) M. J. Bowers, J. R. McBride, and S. J. Rosenthal, J. Am. Chem. Soc., 127, 15378 (2005).

33) H. Wang, A. Tashiro, H. Nakamura, M. Uehara, M. Miyazaki, T. Watari, and H. Maeda, J. Mater. Res., 19, 3157 (2004).

34) Z. A. Peng and X. Peng, J. Am. Chem. Soc., 124, 3343 (2002).

35) S. F. Wuister, F. Van Driel, and A. Meijerink, Phys. Chem. Chem. Phys., 5, 1253 (2003).

36) T. Vossmeyer, L. Katsikas, M. Giersig, I. G. Popovic, K. Diesner, A. Chemseddine, A. Eychmüller, and $\mathrm{H}$. Weller, J. Phys. Chem., 98, 7665 (1994).

37) Y. Nosaka, H. Shigeno, and T. Ikeuchi, J. Phys. Chem., 99, 8317 (1995).

38) A. Hässelbarth, A. Eychmüller, and H. Weller, Chem. Phys. Lett., 203, 271 (1993).

39) T. Rajh, O. I. Micic, and A. J. Nozik, J. Phys. Chem., 97, 11999 (1993).

40) H. Matsumoto, H. Uchida, T. Matsunaga, K. Tanaka, T. Sakata, H. Mori, and H. Yoneyama, J. Phys. Chem., 98, 11549 (1994). 